\title{
A Proper-Orthogonal Decomposition Variational Multiscale Approximation Method for a Generalized Oseen Problem
}

\author{
John Paul Roop \\ Department of Mathematics, North Carolina A \& T State University, Greensboro, NC 27411, USA \\ Correspondence should be addressed to John Paul Roop; jproop@yahoo.com
}

Received 6 June 2013; Accepted 25 October 2013

Academic Editor: Yinnian He

Copyright (C) 2013 John Paul Roop. This is an open access article distributed under the Creative Commons Attribution License, which permits unrestricted use, distribution, and reproduction in any medium, provided the original work is properly cited.

\begin{abstract}
We introduce the variational multiscale (VMS) stabilization for the reduced-order modeling of incompressible flows. It is well known that the proper orthogonal decomposition (POD) technique in reduced-order modeling experiences numerical instability when applied to complex flow problems. In this case a POD discretization naturally separates out structures which corresponding to the energy cascade on large and small scales, in order, a VMS approach is natural. In this paper, we provide the mathematical background necessary for implementing VMS to a POD-Galerkin model of a generalized Oseen problem. We provide theoretical evidence which indicates the consistency of utilizing a VMS approach in the stabilization of reduced order flows. In addition we provide numerical experiments indicating that VMS improves fidelity in reproducing the qualitative properties of the flow.
\end{abstract}

\section{Introduction}

In this paper, we present an analysis and numerical experiments associated with the use of the variational multiscale (VMS) stabilization technique applied to reduced-order modeling (ROM) of a generalized Oseen problem. In producing error estimates and stability results regarding the simulation of incompressible flow problems, the Oseen problem is a model problem in that it possesses all of the features which may prove challenging in the full scale numerical simulation of turbulent flows, while at the same time being posed as a linear problem. In addition, when using an iterative method for resolving the nonlinearity in the incompressible Navier-Stokes equations, an Oseen problem is solved at each iteration.

The generalized Oseen problem may be stated as follows. Let $\Omega$ be a domain in $\mathbb{R}^{\grave{d}}$ (d̀ denoting the spatial dimension), and find $(\mathbf{u}, p): \Omega \rightarrow \mathbb{R}^{\grave{d}} \times \mathbb{R}$ such that

$$
\begin{array}{r}
\partial_{t} \mathbf{u}-\epsilon \Delta \mathbf{u}+\mathbf{b} \cdot \nabla \mathbf{u}+\mathbf{c I} \cdot \mathbf{u}+\nabla p=\mathbf{f} \\
\operatorname{in~}(0, T] \times \Omega \subset \mathbb{R}^{\grave{d}},
\end{array}
$$

$$
\begin{gathered}
\nabla \cdot \mathbf{u}=0 \quad \text { in }(0, T] \times \Omega, \\
\mathbf{u}(\mathbf{x}, 0)=\mathbf{u}_{0}(\mathbf{x}) \quad \text { in } \Omega, \\
\mathbf{u}(\mathbf{x}, t)=0 \quad \text { on }(0, T] \times \partial \Omega .
\end{gathered}
$$

In the case of fluid flow, $\mathbf{u}$ gives the fluid velocity, $p$ is the pressure, $\epsilon \ll 1$ represents the fluid viscosity, $\mathbf{b}$ is a solenoidal vector function, $\nabla \cdot \mathbf{b}=0$, representing the associated velocity field, and $\mathbf{c}$ is a reaction term.

One promising idea when considering the fast simulation of is the use of ROM by way of proper orthogonal decomposition (POD). The idea of applying POD to turbulent flows is nothing new. In fact, the principal components of a flow can effectively give a lot of information about flow's coherent structure and dynamical behavior. However, what remains a mystery is the idea of using the principal components in the fast reproduction of (or variation of modeling parameters for) a fluid flow simulation, which is subject to numerical instability.

The idea behind POD based modeling is nothing new in engineering and is incorporated in many areas. It is quite 
natural to think of a quick model as one which takes steps in the direction of an orthogonal component which maximizes the incorporated variation. However, there are still many unanswered questions as to the underlying mathematical reasons that some flows can be easily reproduced using a POD-Galerkin approach and some cannot. In addition, the nature of POD-Galerkin lends itself to thoughts of nonlinear dynamical systems. For a more general discussion of the way POD-Galerkin is applied and can be analyzed, see [1-3].

In Section 2, we present notations and mathematical background necessary for analyzing the POD-Galerkin problem for an Oseen flow, including specific discussions of how the POD approximation is obtained and the error can be characterized. In Section 3, we introduce the POD-VMS model. In Section 4, we provide an error result which gives the consistency of the POD-VMS model in terms of both the standard finite element error analysis and the error associated with the POD approximation. In Section 5, we provide computational experiments which illustrate the theoretical results as well as the fact that the POD-VMS approach may be utilized to improve the results when applied to a complicated Oseen flow problem. Finally, Section 6 contains conclusions and future work.

\section{Preliminaries}

In this section, we give the appropriate function space setting for the analysis and numerical approximation for the generalized Oseen problem (1). We review some key results which are necessary for the analysis and approximation of (1). Next, we review the VMS method for the standard finite element discretization of (1) and give a discussion of how these may in effect "stabilize" the finite element approximation. We note and will make use of the fact that many of these results carry over to the reduced-order case.

We make use of the standard Sobolev spaces $W^{k, p}(\Omega)$, $H^{k}(\Omega) H_{0}^{k}(\Omega), L^{2}(\Omega)=H^{0}(\Omega)$, and $L_{0}^{2}(\Omega)$ and by utilizing bold face, we mean the same spaces for vector-valued functions. Unless denoted otherwise $\|\cdot\|$ denotes the standard $L^{2}$ norm, $\|\cdot\|_{k}$ the standard $H^{k}$ norm, and $|\cdot|_{k}$ the standard $H^{k}$ seminorm, all taken over $\Omega$. In the sequel, we make use of various spaces, all of which are subspaces of $\mathbf{H}_{0}^{1}(\Omega)$. For abstractness of some definitions, we set $\boldsymbol{\Xi} \subseteq \mathbf{H}_{0}^{1}(\Omega)$.

To facilitate the analysis, we define the following bilinear form for arbitrary $\mathbf{u}, \mathbf{v} \in \boldsymbol{\Xi}$ :

$$
\begin{array}{r}
A(\mathbf{u}, \mathbf{v}):=\epsilon(\nabla \mathbf{u}, \nabla \mathbf{v})+(\mathbf{b} \cdot \nabla \mathbf{u}, \mathbf{v})+(\mathbf{c} \cdot \mathbf{u}, \mathbf{v}) \\
\forall \mathbf{u}, \mathbf{v} \in \mathbf{\Xi} .
\end{array}
$$

We now introduce the variational form for (1) in terms of the previously defined scalar products.

Problem $O$. Find $(\mathbf{u}, p) \in \mathbf{H}_{0}^{1}(\Omega) \times L_{0}^{2}(\Omega)$ s.t.

$$
\begin{array}{r}
\left(\partial_{t} \mathbf{u}, \mathbf{v}\right)+A(\mathbf{u}, \mathbf{v})-(p, \nabla \cdot \mathbf{v})+(q, \nabla \cdot \mathbf{u})=(\mathbf{f}, \mathbf{v}), \\
\forall(\mathbf{v}, q) \in \mathbf{H}_{0}^{1}(\Omega) \times L_{0}^{2}(\Omega) .
\end{array}
$$

Existence and uniqueness first follow from the LaxMilgram lemma, as

$$
(\mathbf{b} \cdot \nabla \mathbf{v}, \mathbf{v})=-\frac{1}{2}(\nabla \cdot \mathbf{b}, \mathbf{v} \cdot \mathbf{v})=0 .
$$

Secondly, a unique pressure in $L_{0}^{2}(\Omega)$ exists from the Babuska-Brezzi condition for the space $\mathbf{H}_{0}^{1}(\Omega) \times L_{0}^{2}(\Omega)$ [4].

We consider conforming finite element spaces $\mathbf{X}_{h}$ and $Q_{h}: \mathbf{X}_{h} \subset \mathbf{H}_{0}^{1}(\Omega), Q_{h} \subset L_{0}^{2}(\Omega)$. We need not be too speci-fic about the error results pertaining to the finite element subspace utilized to provide the underlying approximation. It is sufficient to assume that the true solution's velocity $\mathbf{u}$ belongs to the space $L^{2}\left(0, T ; \mathbf{H}^{m+1}(\Omega)\right)$ and that the finite element approximation affords a Ritz-projection convergence rate of $m+1$ with respect to the $L^{2}(\Omega)$ norm and $m$ with respect to the $H^{1}(\Omega)$ seminorm, respectively.

In the finite element space, we also define the Galerkin approximation.

Problem $\mathrm{OH}$. Find $\left(\mathbf{u}_{h}, p_{h}\right) \in \mathbf{X}_{h} \times Q_{h}$ s.t.

$$
\begin{array}{r}
\left(\partial_{t} \mathbf{u}_{h}, \mathbf{v}_{h}\right)+A\left(\mathbf{u}_{h}, \mathbf{v}_{h}\right)-\left(p_{h}, \nabla \cdot \mathbf{v}_{h}\right)+\left(q_{h}, \nabla \cdot \mathbf{u}_{h}\right)=\left(\mathbf{f}, \mathbf{v}_{h}\right), \\
\forall\left(\mathbf{v}_{h}, q_{h}\right) \in \mathbf{X}_{h} \times Q_{h} .
\end{array}
$$

Problem $\mathrm{OH}$ is guaranteed to possess a unique solution provided that the space $\mathbf{X}_{h} \times Q_{h}$ satisfies the discrete Babuska-Brezzi condition, which implies that the space of weakly divergence-free functions $\mathbf{V}_{h}$,

$$
\mathbf{V}_{h}=\left\{\mathbf{v}_{h} \in \mathbf{X}_{h}:\left(q_{h}, \nabla \cdot \mathbf{v}_{h}\right)=0, \forall q_{h} \in Q_{h}\right\}
$$

is a well-defined, nontrivial, closed subspace of $\mathbf{X}_{h}$ which leads to the fact that the pressure $p_{h}$ is unique [4].

Prior to giving results necessary for the study of the POD discretization of (1), let us briefly review the concepts of VMS and SUPG. The variational multiscale method is a discretization-dependent way in which artificial viscosity is applied in a clever way. Suppose that $\mathbf{X}_{H}$ is at least a partial derefinement of $\mathbf{X}_{h}$; that is, $\mathbf{X}_{H} \subset \mathbf{X}_{h}$. Then we define

$$
V\left(\mathbf{w}_{h}, \mathbf{v}_{h}\right):=\left(P_{H}^{\prime} \nabla \mathbf{w}_{h}, P_{H}^{\prime} \nabla \mathbf{v}_{h}\right),
$$

where $P_{H}$ is the projection operator from $\mathbf{X}_{h}$ onto $\mathbf{X}_{H}$ (which in the case of a Lagrange basis evaluates the interpolant of a function on $\mathbf{X}_{h}$ at the nodes corresponding to the basis in $\mathbf{X}_{H}$ ) and $P_{H}^{\prime}:=\llbracket-P_{H}$.

Problem $\mathrm{OHV}$. Find $\left(\mathbf{u}_{h}, p_{h}\right) \in \mathbf{X}_{h} \times Q_{h}$ s.t.

$$
\begin{array}{r}
\alpha V\left(\mathbf{u}_{h}, \mathbf{v}_{h}\right)+\left(\partial_{t} \mathbf{u}_{h}, \mathbf{v}_{h}\right)+A\left(\mathbf{u}_{h}, \mathbf{v}_{h}\right)-\left(p_{h}, \nabla \cdot \mathbf{v}_{h}\right) \\
+\left(q_{h}, \nabla \cdot \mathbf{u}_{h}\right)=\left(\mathbf{f}, \mathbf{v}_{h}\right), \\
\forall\left(\mathbf{v}_{h}, q_{h}\right) \in \mathbf{X}_{h} \times Q_{h} .
\end{array}
$$

Now that we have established some results regarding the variational solution to the Oseen problem, we introduce the proper orthogonal decomposition. In order to simplify 
the analysis, we consider $\mathbf{u}(\cdot, t)$ as the solution to (3) and consider a finite number of realizations of the solution at various time values. That is, define

$$
\mathscr{H}_{N}:=\operatorname{span}\left\{\mathbf{u}_{h}\left(\cdot, t_{0}\right), \mathbf{u}_{h}\left(\cdot, t_{2}\right), \ldots, \mathbf{u}_{h}\left(\cdot, t_{N}\right)\right\},
$$

where $t_{i}=i T / N, i=0, \ldots, N$. It is effective to think of these "snapshots" as coming from a perfect time discretization of one of the semidiscrete problems. We assume that now a POD decomposition has been performed on the snapshots, which produces an ordered basis:

$$
\mathscr{V}^{r}=\operatorname{span}\left\{\phi_{1}(\cdot), \ldots, \phi_{r}(\cdot)\right\}, \quad 1 \leq r \leq d,
$$

where $d$ is the number of positive singular values of $\mathscr{H}_{N}$ and these values are written in descending order. In addition, we assume that $\left\|\phi_{j}\right\|_{1}=1 \forall 1 \leq j \leq d$, and

$$
\begin{array}{r}
\Lambda_{r}:=\sqrt{\sum_{j=r+1}^{d} \lambda_{j}}=\left\|\mathbf{u}_{h}\left(\cdot, t_{i}\right)-\sum_{j=1}^{r} a_{j} \phi_{j}(\cdot)\right\|_{1}, \\
\forall i=0, \ldots, N,
\end{array}
$$

where $a_{j}:=\left(\mathbf{u}_{h}\left(\cdot, t_{i}\right), \phi_{j}\right), \lambda_{1} \geq \lambda_{2} \geq \cdots \geq \lambda_{d}>0$. We note that the POD-Galerkin truncation is the approximation $u_{r} \in \mathscr{V}^{r}$ of $\mathbf{u}_{h}$ :

$$
\mathbf{u}_{r}(\mathbf{x}, t):=\sum_{j=1}^{r} a_{j}(t) \phi_{j}(\mathbf{x}),
$$

and thus a Ritz-Galerkin approximation is an obvious choice of a test problem in this setting.

\section{The POD-VMS Model}

In this section, we introduce the POD-VMS model in the appropriate function space setting and provide existence, uniqueness, and stability results corresponding to the model.

Problem $P O H V$. Given $R$, find $\mathbf{u}_{r} \in \mathscr{V}^{r}$ s.t.

$$
\begin{array}{r}
\alpha \widetilde{V}\left(\mathbf{u}_{r}, \mathbf{v}_{r}\right)+\left(\partial_{t} \mathbf{u}_{r}, \mathbf{v}_{r}\right)+A\left(\mathbf{u}_{r}, \mathbf{v}_{r}\right)=\left(\mathbf{f}, \mathbf{v}_{r}\right), \\
\forall \mathbf{v}_{r} \in \mathscr{V}^{r} .
\end{array}
$$

We have notationally distinguished the difference between $V$ in the traditional VMS scheme and $\widetilde{V}$ in the POD-VMS scheme as follows:

$$
\widetilde{V}\left(\mathbf{w}_{r}, \mathbf{v}_{r}\right):=\left(P_{R}^{\prime} \nabla \mathbf{w}_{r}, P_{R}^{\prime} \nabla \mathbf{v}_{r}\right),
$$

where $P_{R}^{\prime}:=\rrbracket-P_{R}$.

Instead of considering a projection $P_{H}$ as the projection on a partial derefinement of the grid, we consider the projection $P_{R}$ as the projection of $\mathbf{w}_{r} \in \mathscr{V}_{r}$ onto a subspace $\mathscr{V}_{R} \subset \mathscr{V}_{r}$, where $R<r$. In other words, only nodes which are in the POD-Galerkin basis but correspond to "low energy" are utilized for stabilization.
We make note of the fact that in (13) the equation does not include the pressure terms, as each element of the space $\mathscr{V}^{r}$ is weakly divergence free. Just as in a Chorin-like projection method, the actual pressure in the reduced basis model can be obtained after the fact by solving a Poisson problem using the residual.

\section{Error Analysis}

In this section, we provide the error result and discuss the implication of the error result within the context of practical computational problems.

Theorem 1. The solution of (13) satisfies the following error estimate:

$$
\begin{gathered}
\left\|\mathbf{u}-\mathbf{u}^{r}\right\|_{L^{\infty}\left(0, t ; \mathbf{L}^{2}\right)}+\epsilon\left|\mathbf{u}-\mathbf{u}^{r}\right|_{L^{2}\left(0, t ; \mathbf{H}^{1}\right)} \\
\leq C\left\{h^{m+1}\left\|\mathbf{u}_{t}\right\|_{L^{2}\left(0, t ; \mathbf{H}^{m+1}\right)}+h^{m}\|\mathbf{u}\|_{L^{2}\left(0, t ; \mathbf{H}^{m+1}\right)}\right. \\
\left.\quad+\sqrt{\alpha} \Lambda_{R}+\Lambda_{r}\right\}
\end{gathered}
$$

Proof. We begin the proof by subtracting (13) from (3), testing against arbitrary $\mathbf{v}^{r} \in \mathscr{V}^{r}$, and setting $q=0$. We thus get

$$
\begin{aligned}
&\left(\partial_{t}\left(\mathbf{u}-\mathbf{u}^{r}\right), \mathbf{v}^{r}\right)-\alpha \widetilde{V}\left(\mathbf{u}^{r}, \mathbf{v}^{r}\right)+A\left(\mathbf{u}-\mathbf{u}^{r}, \mathbf{v}^{r}\right)=0, \\
& \forall \mathbf{v}^{r} \in \mathscr{V}^{r} .
\end{aligned}
$$

We decompose the error as follows: $\mathbf{u}-\mathbf{u}^{r}=\boldsymbol{\eta}-\boldsymbol{\phi}^{r}$, where

$$
\boldsymbol{\eta}:=\mathbf{u}-\mathscr{I}^{r} \mathbf{u}, \quad \phi^{r}:=\mathbf{u}^{r}-\mathscr{J}^{r} \mathbf{u},
$$

where $\mathscr{J}^{r} \mathbf{u}$ is the interpolant of $\mathbf{u}$ onto $\mathscr{V}^{r}$, noting that, as we possess an orthogonal basis of $\mathscr{V}^{r}$, a projection thereof is trivial. Also, the interpolating properties of $\boldsymbol{\eta}$ and the error estimate (11) are trivial.

Now, using the definition of $\boldsymbol{\eta}, \boldsymbol{\phi}_{r}$, and (16), we have

$$
\begin{aligned}
&\left(\partial_{t} \boldsymbol{\phi}^{r}, \mathbf{v}^{r}\right)+\alpha \widetilde{V}\left(\boldsymbol{\phi}^{r}, \mathbf{v}^{r}\right)+A\left(\boldsymbol{\phi}^{r}, \mathbf{v}^{r}\right) \\
&=\left(\partial_{t} \boldsymbol{\eta}, \mathbf{v}^{r}\right)-\alpha \widetilde{V}\left(\mathscr{I}^{r} \mathbf{u}, \mathbf{v}^{r}\right)+A\left(\boldsymbol{\eta}, \mathbf{v}^{r}\right), \\
& \forall \mathbf{v}^{r} \in \mathscr{V}^{r} .
\end{aligned}
$$

Setting $\mathbf{v}^{r}=\phi^{r}$, noting the positivity of $\alpha \widetilde{V}\left(\phi^{r}, \phi^{r}\right)$, and using the Cauchy-Schwartz inequality, we have

$$
\begin{aligned}
\left(\partial_{t} \boldsymbol{\phi}^{r}, \boldsymbol{\phi}^{r}\right)+\epsilon\left\|\nabla \boldsymbol{\phi}^{r}\right\|^{2}+\alpha\left\|P_{R}^{\prime} \nabla \boldsymbol{\phi}^{r}\right\|^{2} \\
\leq\left\|\partial_{t} \boldsymbol{\eta}\right\|\left\|\boldsymbol{\phi}^{r}\right\|+\alpha\left\|P_{R}^{\prime} \nabla \mathcal{F}^{r} \mathbf{u}\right\|\left\|P_{R}^{\prime} \nabla \boldsymbol{\phi}^{r}\right\| \\
+\left(\epsilon+C_{c}\right)\|\nabla \boldsymbol{\eta}\|\left\|\nabla \boldsymbol{\phi}^{r}\right\|,
\end{aligned}
$$

where $C_{c}$ is the constant of continuity in the bilinear form. 
Using the fact that $\left(\partial_{t} \phi^{r}, \phi^{r}\right)=(1 / 2)(d / d t)\left\|\phi^{r}\right\|^{2}$ on the left-hand side and Young's inequality twice on the right, we have the following:

$$
\begin{aligned}
\frac{1}{2} \frac{d}{d t}\left\|\phi^{r}\right\|^{2}+\epsilon\left\|\nabla \boldsymbol{\phi}^{r}\right\|^{2}+\alpha\left\|P_{R}^{\prime} \nabla \boldsymbol{\phi}^{r}\right\|^{2} \\
\leq \frac{1}{2}\left\|\partial_{t} \boldsymbol{\eta}\right\|^{2}+\frac{1}{2}\left\|\boldsymbol{\phi}^{r}\right\|^{2} \\
+\frac{\alpha}{2}\left\|P_{R}^{\prime} \nabla \mathscr{J}^{r} \mathbf{u}\right\|^{2}+\frac{\alpha}{2}\left\|P_{R}^{\prime} \nabla \boldsymbol{\phi}^{r}\right\|^{2} \\
+\frac{1}{2 \epsilon}\left(\epsilon+C_{c}\right)\|\nabla \boldsymbol{\eta}\|^{2}+\frac{\epsilon}{2}\left\|\nabla \boldsymbol{\phi}^{r}\right\|^{2} .
\end{aligned}
$$

Now, subtracting through by the appropriate quantities, we have

$$
\begin{aligned}
\frac{1}{2} \frac{d}{d t}\left\|\boldsymbol{\phi}^{r}\right\|^{2}+\frac{\epsilon}{2}\left\|\nabla \boldsymbol{\phi}^{r}\right\|^{2} \\
\leq \frac{1}{2}\left\|\partial_{t} \boldsymbol{\eta}\right\|^{2}+\frac{1}{2}\left\|\boldsymbol{\phi}^{r}\right\|^{2}+\frac{\alpha}{2}\left\|P_{R}^{\prime} \nabla \mathscr{J}^{r} \mathbf{u}\right\|^{2} \\
\quad+\frac{1}{2 \epsilon}\left(\epsilon+C_{c}\right)\|\nabla \boldsymbol{\eta}\|^{2} .
\end{aligned}
$$

Now, multiplying by two, using the fact that $\boldsymbol{\eta}=\left(\mathbf{u}-\mathscr{I}^{h} \mathbf{u}\right)+$ $\left(\mathscr{I}^{h} \mathbf{u}-\mathscr{I}^{r} \mathbf{u}\right)$, where by $\mathscr{I}^{h}$ we mean the projection of $\mathbf{u}$ onto the space $\mathbf{X}_{h}$ and $\mathscr{I}^{r}$ is as defined before, and the triangle inequality, we obtain

$$
\begin{aligned}
\frac{d}{d t}\left\|\boldsymbol{\phi}^{r}\right\|^{2}+\epsilon\left\|\nabla \boldsymbol{\phi}^{r}\right\|^{2} \leq & h^{2 m+2}\left\|\mathbf{u}_{t}\right\|_{H^{m+1}}^{2}+\left\|\boldsymbol{\phi}^{r}\right\|^{2} \\
& +\alpha \Lambda_{R}^{2}+\left(1+\frac{C_{c}}{\epsilon}\right) h^{2 m}\|\mathbf{u}\|_{H^{m+1}}^{2}+\Lambda_{r}^{2} .
\end{aligned}
$$

Finally, an application of Gronwall's inequality yields the desired result.

\section{Computational Experiments}

In this section, we present a number of computational experiments which both support the theoretical estimates obtained above and indicate the improvement achieved by utilizing the VMS stabilization in the POD-ROM approximation. We first present two computational experiments in which the true solution is known and then a third computational experiment with highly heterogeneous flow field in which the true solution is unknown.

First, when considering exact solutions of the Oseen problem (1) (for $d=2)$, the velocity $\mathbf{u}=(u, v)$ must satisfy the continuity equation. However, when resolving reduced-order POD-ROM problems for equations with nonzero essential boundary conditions which change with time, an unnecessary computational challenge is created. There are two main approaches to solving this problem, found in $[5,6]$. Most analytical solutions which are solenoidal possess nonzero

\begin{tabular}{|c|c|c|c|c|c|}
\hline$r$ & $R$ & $\alpha$ & $\left\|\mathbf{u}-\mathbf{u}^{r}\right\|_{0,2}$ & $\left\|\mathbf{u}-\mathbf{u}^{r}\right\|_{1,2}$ & Time (s) \\
\hline 10 & 5 & $h$ & $6.981238 \cdot 10^{-3}$ & $2.422689 \cdot 10^{-1}$ & 811.1 \\
\hline 10 & 8 & $h$ & $6.985801 \cdot 10^{-3}$ & $2.615740 \cdot 10^{-1}$ & 765.6 \\
\hline 10 & 5 & $4 h$ & $6.978340 \cdot 10^{-3}$ & $2.388691 \cdot 10^{-1}$ & 795.7 \\
\hline 10 & 8 & $4 h$ & $6.984956 \cdot 10^{-3}$ & $2.614661 \cdot 10^{-1}$ & 761.9 \\
\hline 10 & 5 & $h / 4$ & $6.987342 \cdot 10^{-3}$ & $2.524964 \cdot 10^{-1}$ & 870.6 \\
\hline 10 & 8 & $h / 4$ & $6.989157 \cdot 10^{-3}$ & $2.612289 \cdot 10^{-1}$ & 836.5 \\
\hline 10 & PODG & N/A & $6.992952 \cdot 10^{-3}$ & $2.597919 \cdot 10^{-1}$ & 698.9 \\
\hline 15 & 5 & $h$ & $6.978064 \cdot 10^{-3}$ & $2.422777 \cdot 10^{-1}$ & 1293.6 \\
\hline 15 & 10 & $h$ & $6.994673 \cdot 10^{-3}$ & $2.594528 \cdot 10^{-1}$ & 1140.7 \\
\hline 15 & 5 & $4 h$ & $6.975261 \cdot 10^{-3}$ & $2.387484 \cdot 10^{-1}$ & 1274.7 \\
\hline 15 & 10 & $4 h$ & $6.992465 \cdot 10^{-3}$ & $2.594345 \cdot 10^{-1}$ & 1114.8 \\
\hline 15 & 5 & $h / 4$ & $6.987577 \cdot 10^{-3}$ & $2.527826 \cdot 10^{-1}$ & 1313.9 \\
\hline 15 & 10 & $h / 4$ & $7.003224 \cdot 10^{-3}$ & $2.600876 \cdot 10^{-1}$ & 1161.4 \\
\hline 15 & PODG & N/A & $7.017659 \cdot 10^{-3}$ & $2.621746 \cdot 10^{-1}$ & 992.6 \\
\hline 20 & 5 & $h$ & $6.979928 \cdot 10^{-3}$ & $2.424063 \cdot 10^{-1}$ & 1999.8 \\
\hline 20 & 10 & $h$ & $6.994185 \cdot 10^{-3}$ & $2.593764 \cdot 10^{-1}$ & 1736.9 \\
\hline 20 & 15 & $h$ & $7.003329 \cdot 10^{-3}$ & $2.610204 \cdot 10^{-1}$ & 1588.1 \\
\hline 20 & 5 & $4 h$ & $6.9757314 \cdot 10^{-3}$ & $2.387778 \cdot 10^{-1}$ & 2014.3 \\
\hline 20 & 10 & $4 h$ & $6.992233 \cdot 10^{-3}$ & $2.593844 \cdot 10^{-1}$ & 1757.9 \\
\hline 20 & 15 & $4 h$ & $7.007108 \cdot 10^{-3}$ & $2.613092 \cdot 10^{-1}$ & 1603.9 \\
\hline 20 & 5 & $h / 4$ & $6.986873 \cdot 10^{-3}$ & $2.526078 \cdot 10^{-1}$ & 2017.9 \\
\hline 20 & 10 & $h / 4$ & $6.998495 \cdot 10^{-3}$ & $2.597420 \cdot 10^{-1}$ & 1762.3 \\
\hline 20 & 15 & $h / 4$ & $7.002788 \cdot 10^{-3}$ & $2.609968 \cdot 10^{-1}$ & 1609.2 \\
\hline 20 & PODG & $\mathrm{N} / \mathrm{A}$ & $7.003565 \cdot 10^{-3}$ & $2.611864 \cdot 10^{-1}$ & 1419.3 \\
\hline 25 & 5 & $h$ & $6.982112 \cdot 10^{-3}$ & $2.426835 \cdot 10^{-1}$ & 2946.9 \\
\hline 25 & 10 & $h$ & $6.993659 \cdot 10^{-3}$ & $2.594056 \cdot 10^{-1}$ & 2683.4 \\
\hline 25 & 15 & $h$ & $7.003330 \cdot 10^{-3}$ & $2.609999 \cdot 10^{-1}$ & 2429.5 \\
\hline 25 & 20 & $h$ & $7.002530 \cdot 10^{-3}$ & $2.611219 \cdot 10^{-1}$ & 2232.8 \\
\hline 25 & 5 & $4 h$ & $6.973241 \cdot 10^{-3}$ & $2.387802 \cdot 10^{-1}$ & 2938.8 \\
\hline 25 & 10 & $4 h$ & $6.989292 \cdot 10^{-3}$ & $2.593093 \cdot 10^{-1}$ & 2588.1 \\
\hline 25 & 15 & $4 h$ & $7.006223 \cdot 10^{-3}$ & $2.612313 \cdot 10^{-1}$ & 2354.9 \\
\hline 25 & 20 & $4 h$ & $7.002944 \cdot 10^{-3}$ & $2.611499 \cdot 10^{-1}$ & 2192.6 \\
\hline 25 & 5 & $h / 4$ & $6.989893 \cdot 10^{-3}$ & $2.528071 \cdot 10^{-1}$ & 3147.3 \\
\hline 25 & 10 & $h / 4$ & $6.999151 \cdot 10^{-3}$ & $2.597719 \cdot 10^{-1}$ & 2762.9 \\
\hline 25 & 15 & $h / 4$ & $7.003222 \cdot 10^{-3}$ & $2.609883 \cdot 10^{-1}$ & 2505.8 \\
\hline 25 & 20 & $h / 4$ & $7.002503 \cdot 10^{-3}$ & $2.611149 \cdot 10^{-1}$ & 2343.9 \\
\hline 25 & PODG & $\mathrm{N} / \mathrm{A}$ & $7.002484 \cdot 10^{-3}$ & $2.611319 \cdot 10^{-1}$ & 2033.2 \\
\hline
\end{tabular}
essential boundary conditions, and most analytical solutions which possess homogeneous boundary conditions are not solenoidal. For a clue about how to construct solutions which are both solenoidal and possess homogeneous boundary
TABLE 1: Comparison of the error between the ROM-calculated velocity $\mathbf{u}^{r}$ and the true solution $\mathbf{u}$ in Experiment 1 for the PODGalerkin approximation and various choices of $R, \alpha$ in the PODVMS model.

conditions, we refer the reader to [7]. It is useful to avoid changing essential boundary conditions, since they have little application in the POD literature anyway.

In the first two experiments, we use solutions whose velocity fields are of the following form:

$$
\begin{gathered}
u=\sum_{k=1}^{n} c_{k}(t) \sin ^{2}(k \pi x) \sin (k \pi y) \cos (k \pi y), \\
v=-\sum_{k=1}^{n} c_{k}(t) \sin (k \pi x) \cos (k \pi x) \sin ^{2}(k \pi y),
\end{gathered}
$$


TABle 2: Comparison of the error between the ROM-calculated velocity $\mathbf{u}^{r}$ and the true solution $\mathbf{u}$ in Experiment 2 for the PODGalerkin approximation and various choices of $R, \alpha$ in the PODVMS model.

\begin{tabular}{|c|c|c|c|c|c|}
\hline$r$ & $R$ & $\alpha$ & $\left\|\mathbf{u}-\mathbf{u}^{r}\right\|_{0,2}$ & $\left\|\mathbf{u}-\mathbf{u}^{r}\right\|_{1,2}$ & Time (s) \\
\hline 10 & 5 & $h$ & $6.442288 \cdot 10^{-3}$ & $2.752475 \cdot 10^{-1}$ & 933.6 \\
\hline 10 & 8 & $h$ & $.882789 \cdot 10^{-3}$ & $1.101335 \cdot 10^{-1}$ & 887.1 \\
\hline 10 & 5 & $4 h$ & $.374255 \cdot 10^{-3}$ & $3.767016 \cdot 10^{-1}$ & 911.2 \\
\hline 10 & 8 & $4 h$ & $.882517 \cdot 10^{-3}$ & $1.092202 \cdot 10^{-1}$ & 863.5 \\
\hline 10 & 5 & $h / 4$ & $4.481848 \cdot 10^{-3}$ & $1.581403 \cdot 10^{-1}$ & 916.0 \\
\hline 10 & 8 & $h / 4$ & $3 \cdot 10^{-3}$ & $1.127396 \cdot 10^{-1}$ & 872.0 \\
\hline 10 & PODG & N/A & $3.883386 \cdot 10^{-3}$ & $1.137251 \cdot 10^{-1}$ & 793.1 \\
\hline 15 & 5 & $h$ & $7 \cdot 10^{-3}$ & $2.718394 \cdot 10^{-1}$ & 1397.7 \\
\hline 15 & 10 & $h$ & $382608 \cdot 10^{-3}$ & $1.136891 \cdot 10^{-1}$ & 1238.9 \\
\hline 15 & 5 & $4 h$ & $8.266850 \cdot 10^{-3}$ & $3.738084 \cdot 10^{-1}$ & 1393.4 \\
\hline 15 & 10 & $4 h$ & $3.883011 \cdot 10^{-3}$ & $1.136693 \cdot 10^{-1}$ & 1238.3 \\
\hline 15 & 5 & $h / 4$ & $4.452304 \cdot 10^{-3}$ & $1.593695 \cdot 10^{-1}$ & 1388.5 \\
\hline 15 & 10 & $h / 4$ & $3.882695 \cdot 10^{-3}$ & $1.142659 \cdot 10^{-1}$ & 1234.5 \\
\hline 15 & PODG & N/A & $3.883556 \cdot 10^{-3}$ & $1.154404 \cdot 10^{-1}$ & 1125.1 \\
\hline 20 & 5 & $h$ & $\cdot 10^{-3}$ & $1 \cdot 10^{-1}$ & 2130.3 \\
\hline 20 & 10 & $h$ & $69 \cdot 10^{-3}$ & $1.136950 \cdot 10^{-1}$ & 1864.1 \\
\hline 20 & 15 & $h$ & & $48 \cdot 10^{-1}$ & \\
\hline 20 & 5 & $4 h$ & $97183 \cdot 10^{-3}$ & $3.713549 \cdot 10^{-1}$ & 2134.3 \\
\hline 20 & 10 & $4 h$ & $5 \cdot 10^{-3}$ & $1.136407 \cdot 10^{-1}$ & 1869.3 \\
\hline 20 & 15 & $4 h$ & $3 \cdot 10^{-3}$ & $518 \cdot 10^{-1}$ & 1709.3 \\
\hline 20 & 5 & $h / 4$ & $51 \cdot 10^{-3}$ & $1.521759 \cdot 10^{-1}$ & 2095.5 \\
\hline 20 & 10 & $h / 4$ & $3.883271 \cdot 10^{-3}$ & $1.143748 \cdot 10^{-1}$ & 1847.3 \\
\hline 20 & 15 & $h / 4$ & $2 \cdot 10^{-3}$ & $.154097 \cdot 10^{-1}$ & 1695.4 \\
\hline 20 & PODG & N/A & $3.884723 \cdot 10^{-3}$ & $1.156279 \cdot 10^{-1}$ & 1593.2 \\
\hline 25 & 5 & $h$ & $6.267525 \cdot 10^{-3}$ & $2.682228 \cdot 10^{-1}$ & 3250.2 \\
\hline 25 & 10 & $h$ & $3.882769 \cdot 10^{-3}$ & $1.137263 \cdot 10^{-1}$ & 2863.5 \\
\hline 25 & 15 & $h$ & $18 \cdot 10^{-3}$ & $1.153711 \cdot 10^{-1}$ & 2590.2 \\
\hline 25 & 20 & $h$ & $7 \cdot 10^{-3}$ & $1.156084 \cdot 10^{-1}$ & 2426.6 \\
\hline 25 & 5 & $4 h$ & $16 \cdot 10^{-3}$ & $3.690778 \cdot 10^{-1}$ & 3212.8 \\
\hline 25 & 10 & $4 h$ & $5 \cdot 10^{-3}$ & $1.136663 \cdot 10^{-1}$ & 2829.7 \\
\hline 25 & 15 & $4 h$ & $3.883670 \cdot 10^{-3}$ & $1.153693 \cdot 10^{-1}$ & 2549.5 \\
\hline 25 & 20 & $4 h$ & $3.334791 \cdot 10^{-3}$ & $1.166200 \cdot 10^{-1}$ & 2391.1 \\
\hline 25 & 5 & $h / 4$ & $4.533282 \cdot 10^{-3}$ & $1.634145 \cdot 10^{-1}$ & 3175.2 \\
\hline 25 & 10 & $h / 4$ & $3.883342 \cdot 10^{-3}$ & $1.143960 \cdot 10^{-1}$ & 2797.1 \\
\hline 25 & 15 & $h / 4$ & $3.884376 \cdot 10^{-3}$ & $1.154101 \cdot 10^{-1}$ & 2468.7 \\
\hline 25 & 20 & $h / 4$ & $3.884735 \cdot 10^{-3}$ & $1.155926 \cdot 10^{-1}$ & 2362.0 \\
\hline 25 & PODG & N/A & $3.884770 \cdot 10^{-3}$ & $1.156054 \cdot 10^{-1}$ & 2184.7 \\
\hline
\end{tabular}

where we clearly see that all choices of $n,\left\{c_{k}(t)\right\}_{k=1}^{n}$ give velocity fields which are solenoidal and possess homogeneous boundary conditions on the unit square.

We now present the results from the three computational experiments. For both Experiments 1 and 2 we consider solutions of the form (23) and use a computational grid with mesh parameter $h=1 / 64$ on the unit square. For all three computational experiments, we set $T=1$ and $\Delta t=0.01$, utilize the Taylor-Hood finite element pair, and solve the problem using $\mathrm{C}++$ on a 12 processor Mac Pro.
TABLE 3: Comparison of the error between the ROM-calculated velocity $\mathbf{u}^{r}$ and the full Galerkin approximation $\mathbf{u}^{h}$ in Experiment 3 for the POD-Galerkin approximation and various choices of $R, \alpha$ in the POD-VMS model.

\begin{tabular}{|c|c|c|c|c|c|}
\hline$r$ & $R$ & $\alpha$ & $\left\|\mathbf{u}^{h}-\mathbf{u}^{r}\right\|_{0,2}$ & $\left\|\mathbf{u}^{h}-\mathbf{u}^{r}\right\|_{1,2}$ & Time (s) \\
\hline 20 & 5 & $h$ & $6.879882 \cdot 10^{-2}$ & 2.291823 & 1366.0 \\
\hline 20 & 10 & $h$ & $7.077484 \cdot 10^{-2}$ & 2.364495 & 1186.8 \\
\hline 20 & 15 & $h$ & $7.366113 \cdot 10^{-2}$ & 2.629697 & 1071.5 \\
\hline 20 & 5 & $4 h$ & $6.855368 \cdot 10^{-2}$ & 2.281231 & 1349.9 \\
\hline 20 & 10 & $4 h$ & $7.092864 \cdot 10^{-2}$ & 2.355982 & 1206.5 \\
\hline 20 & 15 & $4 h$ & $7.368741 \cdot 10^{-2}$ & 2.629806 & 1093.8 \\
\hline 20 & 5 & $h / 4$ & $6.991190 \cdot 10^{-2}$ & 2.365337 & 1358.6 \\
\hline 20 & 10 & $h / 4$ & $7.110597 \cdot 10^{-2}$ & 2.423825 & 1190.6 \\
\hline 20 & 15 & $h / 4$ & $7.363914 \cdot 10^{-2}$ & 2.631096 & 1089.8 \\
\hline 20 & PODG & N/A & $7.331827 \cdot 10^{-2}$ & 2.645780 & 985.5 \\
\hline 25 & 5 & $h$ & $6.883794 \cdot 10^{-2}$ & 2.293204 & 2065.5 \\
\hline 25 & 10 & $h$ & $7.082168 \cdot 10^{-2}$ & 2.365916 & 1805.9 \\
\hline 25 & 15 & $h$ & $7.368019 \cdot 10^{-2}$ & 2.630690 & 1620.3 \\
\hline 25 & 20 & $h$ & $7.371688 \cdot 10^{-2}$ & 2.638875 & 1508.2 \\
\hline 25 & 5 & $4 h$ & $6.856039 \cdot 10^{-2}$ & 2.281288 & 2087.6 \\
\hline 25 & 10 & $4 h$ & $7.093844 \cdot 10^{-2}$ & 2.356012 & 1819.7 \\
\hline 25 & 15 & $4 h$ & $7.368747 \cdot 10^{-2}$ & 2.629795 & 1634.1 \\
\hline 25 & 20 & $4 h$ & $7.369346 \cdot 10^{-2}$ & 2.637818 & 1521.5 \\
\hline 25 & 5 & $h / 4$ & $7.009100 \cdot 10^{-2}$ & 2.375302 & 2084.2 \\
\hline 25 & 10 & $h / 4$ & $7.129551 \cdot 10^{-2}$ & 2.433451 & 1821.5 \\
\hline 25 & 15 & $h / 4$ & $7.378634 \cdot 10^{-2}$ & 2.639321 & 1635.0 \\
\hline 25 & 20 & $h / 4$ & $7.384709 \cdot 10^{-2}$ & 2.645075 & 1525.0 \\
\hline 25 & PODG & N/A & $7.432488 \cdot 10^{-2}$ & 2.676415 & 1411.8 \\
\hline 30 & 5 & $h$ & $6.881790 \cdot 10^{-2}$ & 2.316679 & 2947.2 \\
\hline 30 & 10 & $h$ & $7.069041 \cdot 10^{-2}$ & 2.388068 & 2605.5 \\
\hline 30 & 15 & $h$ & $7.333944 \cdot 10^{-2}$ & 2.645757 & 2337.7 \\
\hline 30 & 20 & $h$ & $7.364633 \cdot 10^{-2}$ & 2.657557 & 2154.8 \\
\hline 30 & 25 & $h$ & $7.415144 \cdot 10^{-2}$ & 2.696152 & 2043.3 \\
\hline 30 & 5 & $4 h$ & $6.856142 \cdot 10^{-2}$ & 2.304418 & 2963.9 \\
\hline 30 & 10 & $4 h$ & $7.080407 \cdot 10^{-2}$ & 2.378906 & 2624.2 \\
\hline 30 & 15 & $4 h$ & $7.332959 \cdot 10^{-2}$ & 2.643993 & 2366.3 \\
\hline 30 & 20 & $4 h$ & $7.342115 \cdot 10^{-2}$ & 2.655854 & 2177.5 \\
\hline 30 & 25 & $4 h$ & $7.414186 \cdot 10^{-2}$ & 2.695809 & 2062.6 \\
\hline 30 & 5 & $h / 4$ & $7.004272 \cdot 10^{-2}$ & 2.400298 & 2962.4 \\
\hline 30 & 10 & $h / 4$ & $7.117844 \cdot 10^{-2}$ & 2.456943 & 2627.9 \\
\hline 30 & 15 & $h / 4$ & $7.352618 \cdot 10^{-2}$ & 2.658138 & 2362.4 \\
\hline 30 & 20 & $h / 4$ & $7.365253 \cdot 10^{-2}$ & 2.667431 & 2177.1 \\
\hline 30 & 25 & $h / 4$ & $7.418287 \cdot 10^{-2}$ & 2.698117 & 2063.2 \\
\hline 30 & PODG & $\mathrm{N} / \mathrm{A}$ & $7.427912 \cdot 10^{-2}$ & 2.705275 & 1950.9 \\
\hline
\end{tabular}

Experiment 1. In this experiment, we choose solutions of the form (23) where $n=5$ and $c_{k}(t)=(1 / k) \cos (k \pi t)$. We use the POD-Galerkin approximation for $r=15,20,25$ and vary both the values of $R$ and $\alpha$ utilized in the POD-VMS model. Experimental error results from this experiment are summarized in Table 1 . We notice that as $R$ is decreased, the error decreases, meaning that the more modes utilized for the VMS stabilization yield a solution which is closer to the true solution. In addition, we see that more damping, that is, using 




O Experiment 1

$\square$ Experiment 2

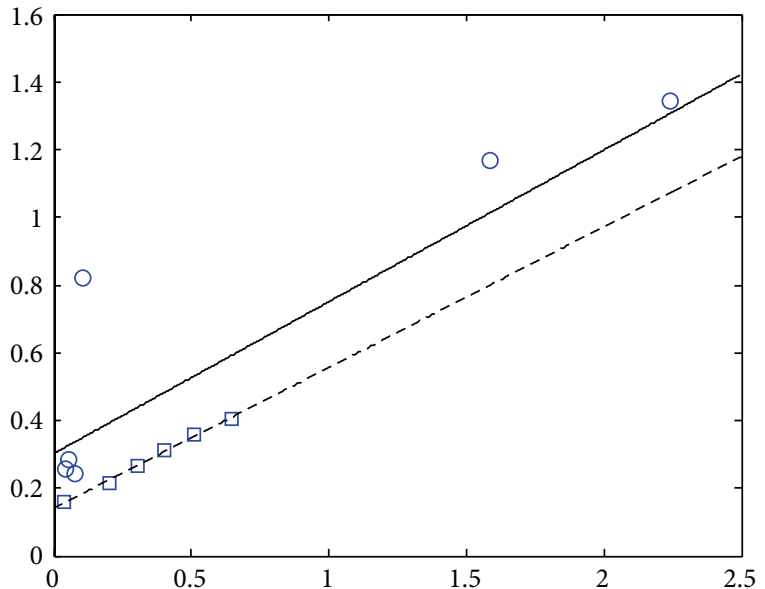

O Experiment 1

Experiment 2

(a)

(b)

Figure 1: (a) Truncation with respect to $R$ versus actual $\mathbf{L}^{2}$ error, for $R=2, \ldots, 7$. (b) Truncation with respect to $R$ versus actual $\mathbf{H}^{1}$ error, for $R=2, \ldots, 7$. Circles are from Experiment 1 . Squares are from Experiment 2 .



(a)


(b)

Figure 2: (a) Horizontal component of b in Experiment 3. (b) Vertical component of b in Experiment 3.

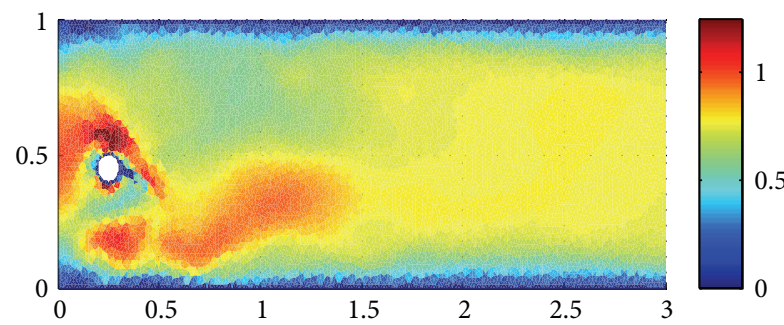

(a)

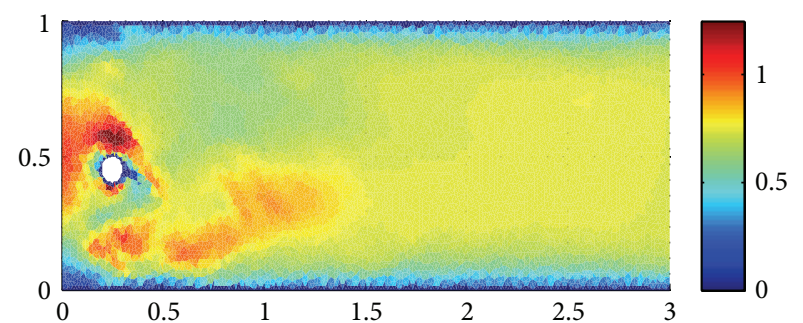

(b)

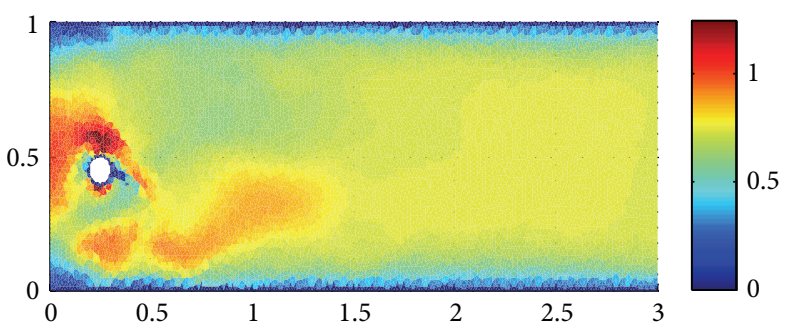

(c)

Figure 3: (a) Horizontal component of the flow $u$ for the DNS simulation in Experiment 3. (b) Horizontal component of the flow $u$ for the POD-Galerkin model with $r=25$. (c) Horizontal component of the flow $u$ for the POD-VMS model with $r=25, R=5$, and $\alpha=4 h$. 


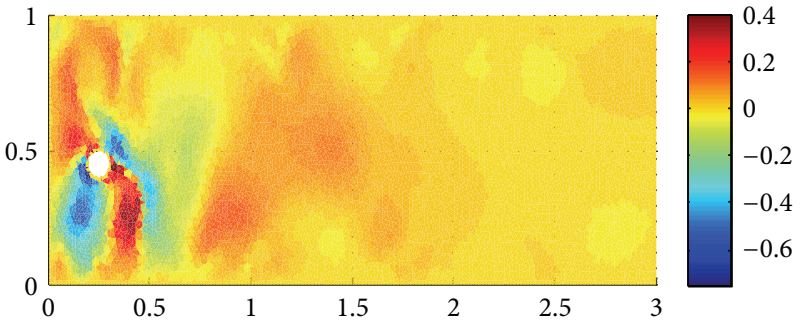

(a)

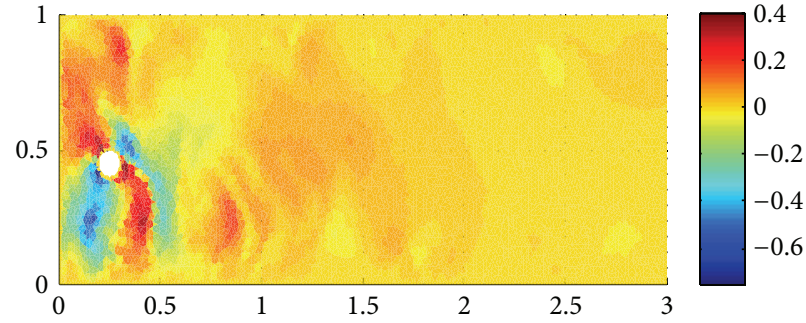

(b)

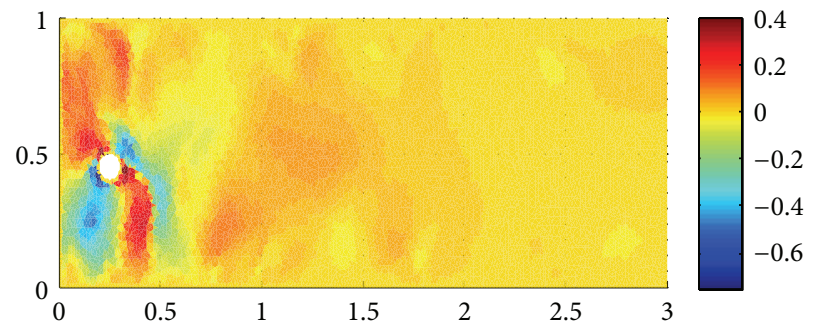

(c)

FIGURE 4: (a) Vertical component of the flow $v$ for the DNS simulation in Experiment 3. (b) Vertical component of the flow $v$ for the PODGalerkin model with $r=25$. (c) Vertical component of the flow $v$ for the POD-VMS model with $r=25, R=5$, and $\alpha=4 h$.

four times $h$ as the modeling parameter $\alpha$, gives a lower error as opposed to using lower values of $\alpha$.

Experiment 2. In this experiment, we choose solutions of the form (23) where $n=8$ and $c_{k}(t)=\left(1 / k^{2}\right) \cos (k \pi t)$. We use the POD-Galerkin approximation for $r=15,20,25$ and vary both the values of $R$ and $\alpha$ utilized in the PODVMS model. Experimental error results from this experiment are summarized in Table 2. In this experiment, we have the opposite behavior from Experiment 1 . We notice that as $R$ is decreased, the error increases, meaning that the less modes utilized for the VMS stabilization yield a solution which is closer to the true solution. In addition, we see that less damping, that is, using one-fourth times $h$ as the modeling parameter $\alpha$, gives a lower error as opposed to using lower values of $\alpha$.

This pattern is further illustrated in Figure 1. Figure 1(a) gives the way that the $\mathbf{L}^{2}$ error varies with $R$ and Figure 1(b) gives the way that $\mathbf{H}^{1}$ error varies with $R$. Even though the number of coefficients is increased from Experiment 1 to Experiment 2 it is clear that the way that the energy decays has more to do with the need for damping in the POD-VMS model. In Experiment 2, the energy decreases quadratically, and thus the POD-Galerkin approximation gives a reasonable approximation with no damping, and so damping only serves to pollute the solution with an unnecessary approximation error. However, in Experiment 1, the energy decreases only linearly and thus the POD-Galerkin error is improved using VMS damping, and the more damping that is applied to the approximation, the better the results. Thus although we can conclude a consistent approximation when using increasing values of $r$ and decreasing values of $\alpha$, we cannot conclude at this time whether there is any optimal way to choose the modeling parameters which might minimize the error of approximation.

Experiment 3. In this experiment, we consider a generalized Oseen problem with Reynolds number 300 (effective viscosity 1/300) where the solenoidal vector field $\mathbf{b}$ is a highly heterogeneous and investigate the ability of VMS stabilization in improving the POD-Galerkin approximation. Each of the components of the field $\mathbf{b}$ was illustrated in Figure 2 and was arrived at using a separate computational code and stored for use at each time step. The underlying mesh utilized for this experiment possesses a grid parameter of $h=1 / 32$ and 24,168 degrees of freedom.

Table 3 gives the experimental error results for this problem. Notice that we cannot utilize $\left\|\mathbf{u}-\mathbf{u}_{r}\right\|$ as the exact solution is unknown; thus we only measure the difference between the POD VMS approximation and the underlying finite element approximation. We see for this experiment that using more modes for the stabilization (low $R$ ) and a higher value of $\alpha$ gives results which are closer to the underlying finite element approximation, thus indicating that a POD VMS stabilization improves the reduced-order approximation for the problem.

Figures 3 and 4 visually illustrate the improvement obtained by VMS stabilization. Each represents the $\mathbf{u}$ and $\mathbf{v}$ components of the flow, respectively, and in each figure, (a) gives the result (at time $T=1$ ) for the underling "DNS," (b) gives the result from the POD-Galerkin approximation and (c) gives the result from the POD-VMS approximation, with $\alpha=4 h$. We see in this example the improvement made to the resulting flow using the VMS stabilization. 


\section{Conclusion}

We have seen the theoretical and computational structure in which the POD-Galerkin method may be applied to a generalized Oseen problem. In addition, we have applied a VMS stabilization and shown that error estimates can be obtained as well that a complex flow can be reproduced. For future work, stabilization methods can be utilized on particular kinds of complex flows, noting that what is usually performed by researchers are computational tricks which tend to indicate improvement for simplistic examples. In particular, we are interested in understanding what occurs dynamically in a POD-Galerkin approximation for flows that are actually complicated dynamically and how POD-Galerkin can be improved using a Petrov-Galerkin approximation.

\section{Acknowledgment}

This research is partially supported by NSF Grant DMS1016591.

\section{References}

[1] J. Borggaard, A. Duggleby, A. Hay, T. Iliescu, and Z. Wang, "Reduced-order modeling of turbulent flows," in Proceedings of the 18th International Symposium on Mathematical Theory of Networks and Systems (MTNS '08), 2008.

[2] T. Iliescu and Z. Wang, "Variational multiscale proper orthogonal decomposition: convection-dominated convection-diffusion-reaction equations," Mathematics of Computation, vol. 82, no. 283, pp. 1357-1378, 2013.

[3] T. Iliescu and Z. Wang, "Variational multiscale proper orthogonal decomposition: Navier-Stokes equations," Numerical Methods for Partial Differential Equations. In press.

[4] V. Girault and P.-A. Raviart, Finite Element Methods for NavierStokes Equations, vol. 5, Springer, Berlin, Germany, 1986.

[5] J. Burkardt, M. Gunzburger, and H.-C. Lee, "POD and CVTbased reduced-order modeling of Navier-Stokes flows," Computer Methods in Applied Mechanics and Engineering, vol. 196, no. 1-3, pp. 337-355, 2006.

[6] M. D. Gunzburger, J. S. Peterson, and J. N. Shadid, "Reducedorder modeling of time-dependent PDEs with multiple parameters in the boundary data," Computer Methods in Applied Mechanics and Engineering, vol. 196, no. 4-6, pp. 1030-1047, 2007.

[7] J. Wang, Y. Wang, and X. Ye, "A robust numerical method for Stokes equations based on divergence-free $H($ div $)$ finite element methods," SIAM Journal on Scientific Computing, vol. 31, no. 4, pp. 2784-2802, 2009. 




Advances in

Operations Research

mansans

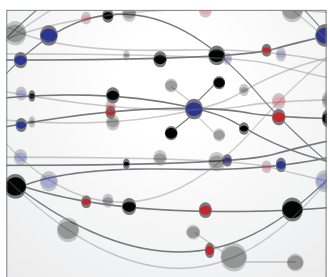

The Scientific World Journal
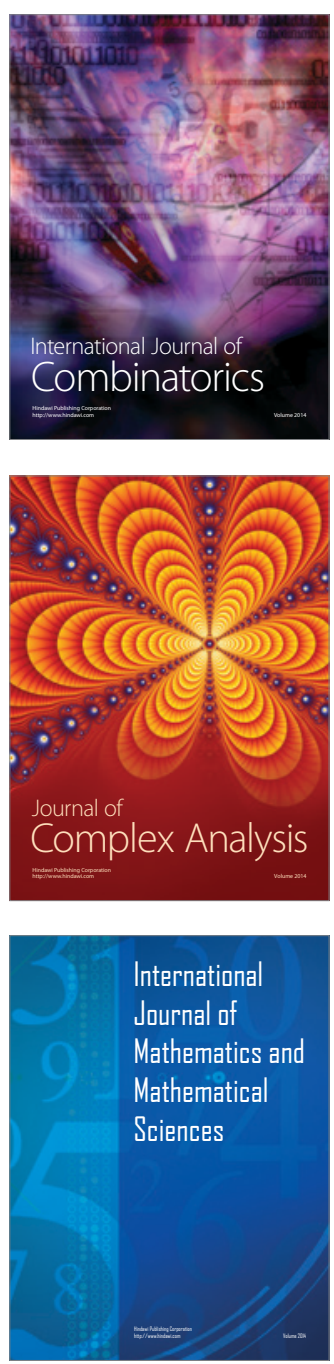
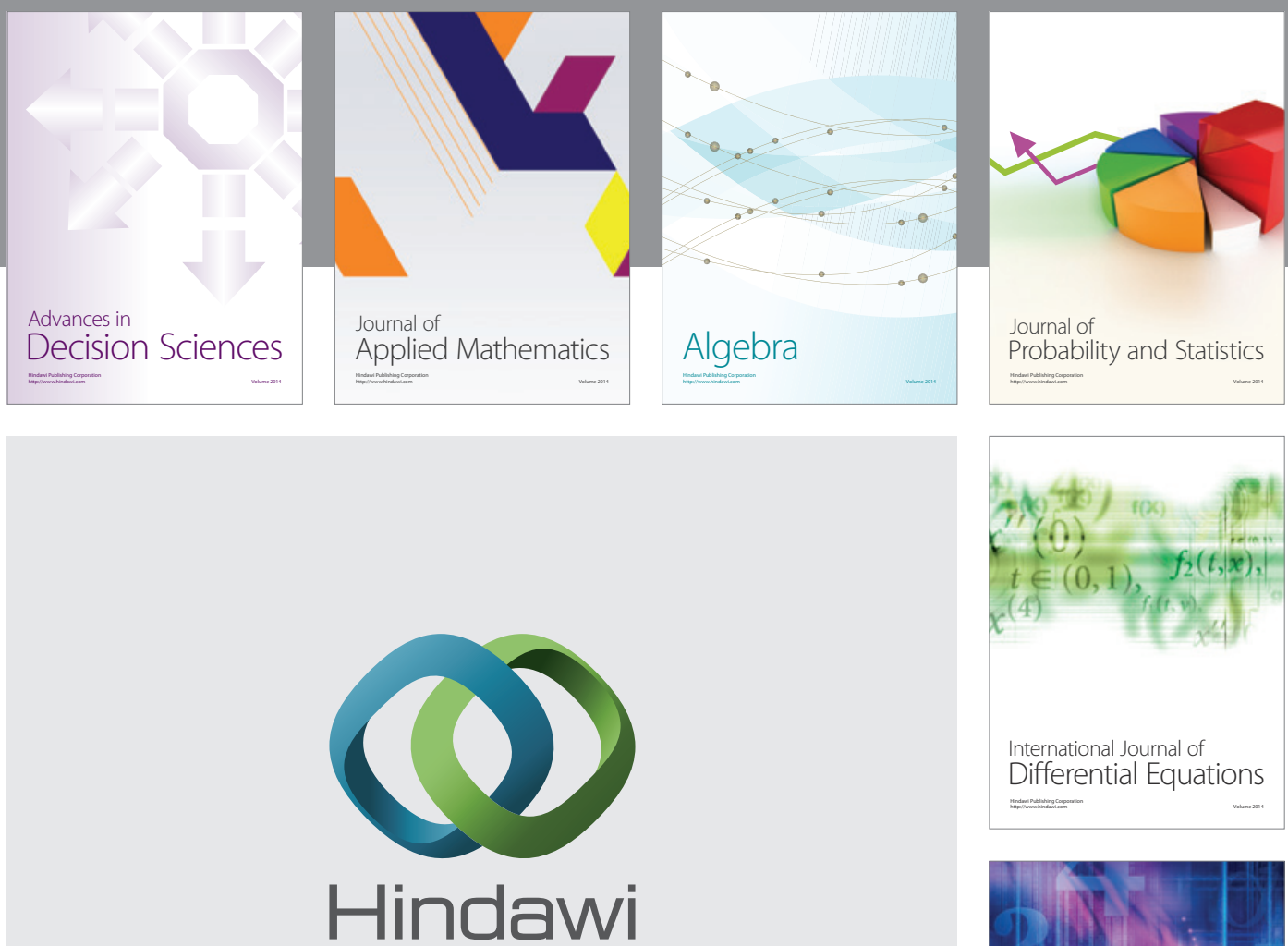

Submit your manuscripts at http://www.hindawi.com
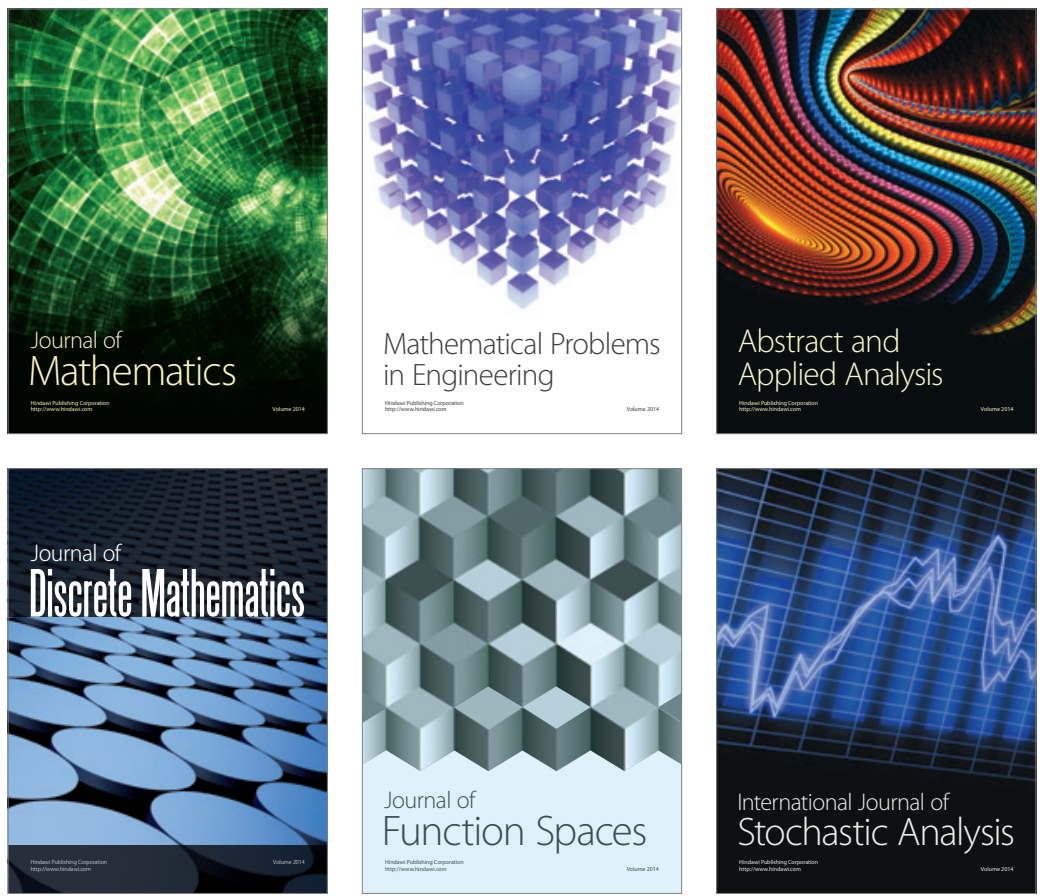

Journal of

Function Spaces



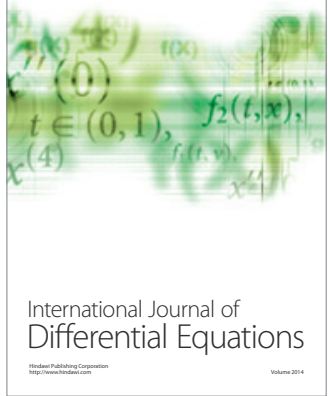
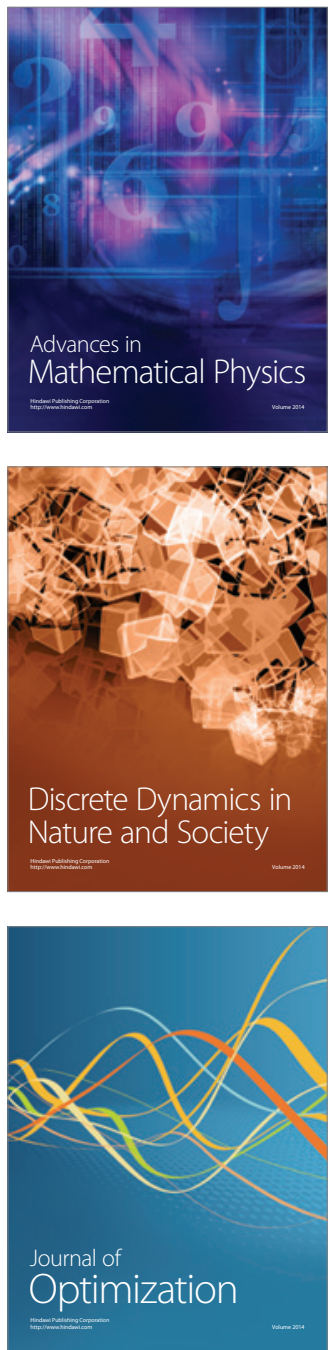